\title{
Digitalisierung und Pflege
}

\author{
Uwe Fachinger und Mareike Mähs \\ (c) Der/die Autor(en) 2019 \\ J. Klauber et al. (Hrsg.), Krankenhaus-Report 2019 \\ https://doi.org/10.1007/978-3-662-58225-1_9
}

\section{Zusammenfassung}

In deutschen Krankenhäusern ergeben sich Anpassungserfordernisse hinsichtlich der Pflege zur Bewältigung der zahlreichen Herausforderungen, zu denen die Zunahme der absoluten Zahl an älteren Menschen, die Veränderungen der Beschäftigtenstruktur, die Arbeitsbedingungen in der Pflege wie auch der Prozess der Digitalisierung gehören. Vor diesem Hintergrund wird digitalen Techniken das Potenzial zugesprochen, die Situation in der Krankenhauspflege verbessern zu können. Es zeigt sich, dass digitale Technologien bereits zur Dokumentation, Informationsverarbeitung und Organisation sowie Kommunikation zwischen den professionellen Pflegekräften eingesetzt werden. Es wird davon ausgegangen, dass weitere Informations- und Kommunikationstechnologien, Roboter und assistierende Technologien in den nächsten Jahren vermehrt in Krankenhäusern eingesetzt werden. Die zunehmende Nutzung derartiger Technologien hat Auswirkungen auf die Arbeitsorganisation, das Berufsbild und das Selbstverständnis der Pflege.

An increasing number of older people, changes in the workforce structure and working conditions in addition to the intensifying process of digitalisation lead to various challenges that require adjustments in inpatient care in German hospitals. Against this background, digital technologies are considered to have the potential to improve inpatient care. They are already being used for documentation, information processing and organisation as well as communication between nurses. It is anticipated that new information and communication technologies, robots and assisting technologies will be used more and more in hospitals. The increasing usage of these technologies will have a strong impact on working conditions, job descriptions and the job related selfperception of caregivers.

\subsection{Einführung}

Die Digitalisierung ist ein mittlerweile alle Lebensbereiche umfassender Prozess und es wird davon ausgegangen, dass die weitere Entwicklung der Arbeitswelt maßgeblich von ihr beeinflusst wird. Die direkten und indirekten Effekte der Digitalisierung auf die Arbeitswelt sind u. a. unter dem Stichwort Arbeitswelt 4.0 intensiv diskutiert worden (Bundesministerium für Arbeit und Soziales 2017; Apt et al. 2016; Schröder und Urban 2016) und es wird davon ausgegangen, dass auch die Pflegetätigkeit in Krankenhäusern von der Digitalisierung direkt und indirekt beeinflusst wird (Daum 2017;
Wibbeling et al. 2017; Fuchs-Frohnhofen et al. 2018). Neben diesen allein durch die technische Entwicklung bedingten Veränderungspotenzialen ergeben sich in deutschen Krankenhäusern Anpassungserfordernisse hinsichtlich der Pflege, um die zahlreichen Herausforderungen zu bewältigen, denen durch den Einsatz digitaler Techniken begegnet werden kann (Evans et al. 2018; Bräutigam et al. 2017; Dörries et al. 2017). Hierzu gehören Veränderungen der Beschäftigtenstruktur wie auch die Arbeitsbedingungen in der Pflege. So werden zum Beispiel Arbeitsintensität und Zeitnot als Probleme in der Pflege genannt, die es nur bedingt zulassen, die Pflegebedürftigen adäquat zu versorgen oder 
auch präventive, aktivierende Behandlungen auszuführen (Deutsche Stiftung Patientenschutz 2018, S. 2; Hien 2017, S. 75; Bruyneel et al. 2015; Ausserhofer et al. 2014; Griffiths et al. 2014; Zander et al. 2014; Sachverständigenrat zur Begutachtung der Entwicklung im Gesundheitswesen 2012, S. 89). Aber auch der Gesundheitsschutz der Pflegekräfte selbst kann durch den Einsatz assistierender Techniken verbessert werden. Aufgrund u. a. von Unterbesetzung, Zeitnot und körperlichen sowie psychischen Belastungen können sich stressbedingte Beschwerden und Muskel-Skelett-Erkrankungen bei den Pflegekräften einstellen und zu krankheitsbedingten Fehltagen sowie einem frühen Austritt aus dem Beruf führen (Dall'Ora et al. 2015; Leineweber et al. 2014; Hämel und Schaeffer 2013, S. 424 f.; Sowinski et al. 2013, S. 42; vgl. Evans et al. 2018, S. 6; Müller 2009; Grabbe et al. 2005).

Eine weitere Herausforderung stellt die $\mathrm{Zu}$ nahme der absoluten Zahl an älteren Menschen dar. Diese hat u. a. zur Folge, dass aufgrund von altersassoziierten Erkrankungen mehr Patienten im Krankenhaus zu versorgen sind (Rösler et al. 2018, S. 7 f.; Hämel und Schaeffer 2013, S. 414). In diesem Zusammenhang ist auch mit einem Anstieg an Patienten mit Multimorbidität und demenziellen Erkrankungen zu rechnen, die einen höheren Arbeitsund Betreuungsaufwand bedingen (Brüggemann et al. 2017; Simon 2015, S. 26 f.). So wird konstatiert, dass in den Krankenhäusern das Pflegepersonal unterbesetzt sei (Bundesregierung 2018; Brüggemann et al. 2017) und dass der Fachkräftemangel in der Pflege sich in Zukunft durch eine steigende Anzahl an Pflegebedürftigen verstärken kann (Augurzky et al. 2018; Evans et al. 2018, S. 6; Rösler et al. 2018, S. 7 f.; Simon 2015, S. 36; Hämel und Schaeffer 2013, S. 418). Eine personelle Unterausstattung sowie eine geringe Qualifikation der Pflegekräfte wirken sich negativ auf die Versorgungsqualität im Krankenhaus aus und können die Mortalität von Patienten erhöhen (Simon 2015, S. 22 ff.; Hämel und Schaeffer 2013, S. 418).

Digitale Techniken haben das Potenzial, den oben kurz skizzierten Herausforderungen in der klinischen Pflege zu begegnen und die Situation der Pflegekräfte selbst zu verbessern (Rösler et al. 2018, S. 9; Roland Berger GmbH et al. 2017, S. 17; Bendig et al. 2017, S. 6). So wird davon ausgegangen, dass durch den Einsatz von Assistenztechnologien eine bessere Pflegequalität erreicht werden und Pflege effizienter sowie effektiver erfolgen kann (Roland Berger GmbH et al. 2017, S. 17; Bendig et al. 2017, S. 17; Daum 2017, S. 33). Um Potenziale digitaler Technik zu identifizieren, ist es wichtig, die Auswirkungen dieser Technologien auf die Strukturen und Rolle der Pflegekräfte im Krankenhaus zu analysieren (vgl. Daum 2017, S. 48; vgl. Bräutigam et al. 2017, S. 11). Der vorliegende Beitrag hat deshalb zum Ziel, im ersten Teil den derzeitigen Stand und zukünftige Entwicklungstendenzen des Einsatzes digitaler Technologien in der Krankenhauspflege darzustellen und, darauf aufbauend, im zweiten Teil die Auswirkungen des Einsatzes derartiger Technologien auf die Arbeitsorganisation, das Berufsbild und das Selbstverständnis der Pflege im Krankenhaus zu diskutieren.

\subsection{Einsatz digitaler Technik in der Krankenhauspflege}

Die Techniken, die in der akutstationären Krankenhauspflege eingesetzt werden, lassen sich grob in Informations- und Kommunikationstechnologien (IKT), Robotik und Assistenztechnologien einteilen, wobei die Grenzen zwischen den einzelnen Systemen - gerade wenn diese zu einem Gesamtsystem vernetzt sind - fließend sind. Im Folgenden werden diese Technologien näher beschrieben und ihr Verbreitungsgrad in der Krankenhauspflege thematisiert.

\subsubsection{Informations- und Kommunikationstechnologien}

Unter dem Begriff IKT können zum einen die elektronische Dokumentation sowie digitale Kommunikationsmittel und zum anderen der Bereich Telemedizin subsumiert werden. Die elektronische Dokumentation ist von den hier vorgestellten Technologien in der akutstationären Versorgung am weitesten verbreitet (Hübner et al. 2018; Hielscher 2014, S. 18 f.; Merda et al. 2018, S. 90 ff.; vgl. Bräutigam et al. 2017, S. 38). Sie dient der Informationsbeschaffung und der Kommunikation zwischen 
allen am Pflegeprozess beteiligten Akteuren (Bundesverband Pflegemanagement 2017; Ball et al. 2011). Die durch die Dokumentation gewonnenen Daten können genutzt werden, um die medizinische und pflegerische Versorgung zu verbessern und allgemein die Prozesse im Krankenhaus zu optimieren, z. B. die Dienstplanung des pflegerischen Personals oder das Patientenmanagement (Mildner et al. 2017, S. 10; Hielscher 2014, S. 18 f.; Hübner et al. 2015, S. 27). Je nach Anwendungszweck kommen unterschiedliche Systeme zum Einsatz. So dient ein Krankenhausinformationssystem (KIS) allgemein der Administration, der Dokumentation und Steuerung von Arbeitsprozessen und als Informationsaustauschplattform aller Akteure aus Verwaltung, Versorgung und Leitung (Bräutigam et al. 2017, S. 14; Mildner et al. 2017, S. 10). So nutzt ein KIS beispielsweise die elektronische Patientenakte, um Patientendaten zu erfassen, zu verwalten und bei Bedarf orts- und zeitunabhängig zur Verfügung zu stellen (Hübner et al. 2018; Frick und Baumberger 2018; Bräutigam et al. 2017, S. 14; Mildner et al. 2017, S. 11).

Spezialisierte Systeme, die mit dem KIS vernetzt oder eigenständig im Krankenhaus zur Anwendung kommen können, sind u. a. die OP-, Labor-, Radiologie-, Intensiv- und Anästhesiedokumentation sowie übergreifende Systeme wie das sogenannte PACS (Picture Archiving and Communication System), die Bilddateien speichern und austauschen können, oder auch Systeme zur Medikamenten-, Hygiene- und Wunddokumentation (Bräutigam et al. 2017, S. 14; Engelmann und Schwind 2017; vgl. Hübner et al. 2015, S. 28 ff.). Zudem gibt es spezialisierte Systeme für die elektronische Pflegedokumentation im Krankenhaus, die der gesetzlich vorgeschriebenen Dokumentation des Pflegeprozesses, der rechtlichen Absicherung, der Kommunikation untereinander und mit anderen Professionen sowie der Qualitätssicherung dienen (Hübner et al. 2015, S. 47; Hielscher 2014, S.18 f.).

Die Systeme sind allerdings auf die interne Prozesssteuerung bzw. das -management ausgerichtet und optimiert. Für die Kommunikation mit externen Dienstleistern - seien es Fach- oder Allgemeinmediziner, andere Kliniken, Rehabilitationseinrichtungen oder ambulante Pflegedienste - zum einrichtungs- und sektorübergreifenden Austausch der Daten, z. B. im Rahmen des Entlassmanagements, sind diese Systeme in der Regel nicht geeignet (Haase 2018; vgl. zur aktuellen Situation Hübner et al. 2018). Es fehlt zurzeit noch an Standards und Maßnahmen, die eine höhere Interoperabilität der unterschiedlichen Dokumentationssysteme gewähren (Mildner et al. 2017, S. 10 ff.; vgl. Hübner et al. 2015, S. 56; Bräutigam et al. 2017, S. 13 f.). Es gibt wenige Ausnahmen hiervon - so u. a. die sogenannten Radiologienetzwerke (Engelmann und Schwind 2017)-, liegen hier doch entsprechende Normen vor, wie die DIN 6868-157 für medizinische Bildwiedergabegeräte (Madsack und Walz 2014).

Prinzipiell können telemedizinische Geräte zum unmittelbaren Austausch von Bild-, Videound Textdaten genutzt werden, um mit Akteuren zu kommunizieren, die sich z. B. auf einer anderen Station, in einem anderen Krankenhaus oder in einer anderen medizinischen Einrichtung befinden (Mildner et al. 2017, S. 27; Dahl und Boulos 2013, S. 14). Denkbar wäre hier beispielsweise, dass sich eine Pflegekraft bei Fragen an eine Kollegin oder einen Kollegen, die Pflegedienstleitung oder auch an das ärztliche Personal wendet und diese Person gegebenenfalls per Videotelefonie zugeschaltet wird (vgl. Vincent und Creteur 2017, S. 2). So wäre es prinzipiell auch möglich, dass der Patient nach der Entlassung in seiner häuslichen Umgebung weiter vom Krankenhauspersonal medizinisch versorgt wird und dieses per Videotelefonie für die pflegebedürftige Person, den ambulanten Pflegedienst oder auch die Hausärzte aus der Distanz heraus beratend zur Verfügung steht (Vincent und Creteur 2017, S. 3; vgl. van den Berg et al. 2015, S. 368). Mit einer derartigen Maßnahme kann möglicherweise nicht nur die Verweildauer verkürzt, sondern auch die Wahrscheinlichkeit der Wiedereinweisung reduziert, die Adhärenz erhöht sowie die Rehabilitation unterstützt, die Versorgung in ländlichen, strukturschwachen Gebieten aufrechterhalten und es können Behandlungsbrüche aufgrund von Sektorenübergängen verhindert werden (Vincent und Creteur 2017, S. 3; vgl. Mildner et al. 2017, S. 27; van den Berg et al. 2015, S. 368).

In Zukunft können potenziell tragbare Geräte zur Speicherung und zum Austausch von Daten, wie die sogenannten Wearables, vermehrt Einzug in die Krankenhauspflege halten (Daum 2017, S. 31; 
vgl. Bräutigam et al. 2017, S. 38). Mithilfe von Tablet-PCs, Smartphones oder Smartwatches können medizinische und pflegerische Daten direkt und unmittelbar in der Nähe des Patienten über das Internet abgerufen, erhoben und versendet werden (Daum 2017, S. 21; vgl. Vincent und Creteur 2017, S. 2; Mildner et al. 2017, S. 26 f.). Diese mobilen Geräte können zusätzlich leitlinienkonforme Arbeitsabläufe vorgeben, Notfälle anzeigen und an bestimmte Arbeitsschritte wie die Medikamentengabe erinnern oder die Notwendigkeit der Flüssigkeitsund Nahrungsaufnahme anzeigen und somit die Pflegekräfte in ihrer Entscheidungsfindung unterstützen (Daum 2017, S. 21; Hübner et al. 2015, S. 34; Mildner et al. 2017, S. 26). Auch wird an einer intelligenten, sensorbasierten Berufskleidung geforscht, mit deren Hilfe Bewegungen, die Körperhaltung und Vitalparameter erfasst werden können, z. B. um die körperliche Belastung für präventive Maßnahmen oder den Pflegeprozess anhand der Bewegungen automatisiert zu dokumentieren (Daum 2017, S. 22).

Smartphones und -watches eignen sich auch zur Kommunikation zwischen den verschiedenen Pflegeprofessionen. So werden private Geräte heute schon zur Dienstplanung (z. B. mithilfe der sozialen Medien) eingesetzt, beispielsweise wenn eine Pflegekraft spontan ausfällt und schnellstmöglich ersetzt werden muss (Bräutigam et al. 2017, S. 27 ff.). Digitale Plattformen können die Kommunikation zwischen den Gesundheitsprofessionen im Krankenhaus standardisieren und somit effizienter gestalten, wenn beispielsweise statt mehrerer Geräte nur noch ein Medium für Kontaktversuche von Personal genutzt wird und gleichzeitig Informationen zur Präsenz und Abwesenheit des Personals eingesehen werden können (Mildner et al. 2017, S. 28)

\subsubsection{Robotik}

Im Bereich Robotik werden Serviceroboter, Behandlungsroboter und sozial-interaktive Roboter hinsichtlich eines Einsatzes im Krankenhaus diskutiert. Die meisten Roboter stellen derzeit noch Prototypen aus Forschungsprojekten dar, $\mathrm{u}$. a. weil die Anforderungen an die Sicherheit und Verlässlich- keit in der Pflege hoch sind (Klein 2011, S. 87; vgl. Daum 2017, S. 26; Graf et al. 2013, S. 1151). Auch wird davon ausgegangen, dass Roboter, wenn überhaupt, noch lange nicht in der Lage sein werden, eine direkte (Bezugs-)Pflege autonom durchzuführen (Daum 2017, S. 23; vgl. Merda et al. 2018, S. 86).

Die zurzeit noch stärkste Nutzung erfahren Serviceroboter. Diese werden in Krankenhäusern zur autonomen Beförderung von Lasten eingesetzt und transportieren u. a. Lebensmittel, Verbrauchsmaterialien, Medikamente, Wäsche und Abfälle (Daum 2017, S. 23; Vincent und Creteur 2017, S. 2; Graf et al. 2013, S. 1149; Dahl und Boulos 2013, S. 4). Erst in wenigen Krankenhäusern werden die Roboter auch in den öffentlichen Stationen eingesetzt und können mithilfe von Sensoren eigenständig navigieren und auch Personen erkennen (Daum 2017, S. 23; Graf et al. 2013, S. 1149; vgl. Dahl und Boulos 2013, S. 4; vgl. Klein und Cook 2009, S. 24). In derartige Roboter können zusätzlich MonitoringTechnologien integriert sein, sodass die Roboter die Flure überwachen und beispielsweise bei der Detektion eines (gestürzten) Patienten die Pflegekraft entsprechend informieren (Daum 2017, S. 23; Graf et al. 2013, S. 1.150).

Weniger bis gar nicht verbreitet sind Roboter, die pflegenahe Tätigkeiten wie Bring- und Holdienste sowie das Heben, Tragen, Lagern und Waschen von Patienten übernehmen können (Graf et al. 2018; Daum 2017, S. 24 ff.). So werden in Forschungsprojekten Roboter entwickelt, die dem Patienten Nahrung, Getränke sowie Medikamente bringen, z. T. anreichen und die Aufnahme dieser kontrollieren können (Klein 2011, S. 87; Daum 2017, S. 26; Vincent und Creteur 2017, S. 2; Graf et al. 2013, S. 1150; Dahl und Boulos 2013, S. 4). Weitere derartige Roboter sind Lifter oder auch intelligente Rollstühle, die Patienten (teil-)automatisiert befördern (Daum 2017, S. 24; Dahl und Boulos 2013, S. 4).

Teilweise kommen in mehreren Krankenhäusern bereits Roboter in Operationssälen zur Anwendung, um Operationen präziser und sicherer durchzuführen (Bräutigam et al. 2017, S. 14; Dahl und Boulos 2013, S. 2; Mildner et al. 2017, S. 19). Zukünftig kann das ärztliche Personal zusätzlich aus der Distanz heraus Operationen mithilfe dieser und telemedizinischer Technologien durchführen 
(Vincent und Creteur 2017, S. 2; Dahl und Boulos 2013, S. 2). Auch wenn primär das ärztliche Personal diese Technologie nutzt, sollten auch die im Operationssaal arbeitenden bzw. bei der Operation unterstützenden Pflegekräfte Kenntnisse über deren Anwendung haben, um dem ärztlichen Personal kompetent assistieren zu können.

Sozial-interaktive Roboter, die mit den Pflegekräften und Patienten interagieren können, befinden sich zum Großteil noch in der Entwicklung (vgl. Daum 2017, S. 25 ff.). Viele dieser Roboter können potenziell auf Stimme, Gestik und Mimik reagieren und diese eingeschränkt auch selbst zeigen (vgl. Daum 2017, S. 25). Sozial-interaktive Roboter können in folgenden Anwendungsgebieten eingesetzt werden: Zum einen können sie als Orientierungshilfe dienen und Patienten wie Besuchern Auskunft erteilen und diese über das Krankenhausgelände zum gewünschten Zimmer/Ort führen (vgl. Daum 2017, S. 25). Zum anderen wären sie für therapeutische Zwecke einsetzbar, z. B. zur Ansprache, Stimulation und Beruhigung von demenziell Erkrankten oder auch von Kindern (vgl. Daum 2017, S. 26 f.; vgl. Dahl und Boulos 2013, S. 7). In Abhängigkeit von der weiteren Entwicklung werden derartige Technologien möglicherweise auch verstärkt in der Krankenpflege z. B. in der Geriatrie nachgefragt werden.

\subsubsection{Assistenztechnologien}

Assistenztechnologien in ihren unterschiedlichen Formen können die Arbeit der Pflegekräfte erleichtern und, in Abgrenzung zu traditionellen Hilfsmitteln, bis zu einem bestimmten Grad autonom agieren.

Telemonitoring kann mit unterschiedlichen Sensoren z. B. erfassen, ob eine Person gestürzt ist, die Station verlässt, es kann Vitalparameter und die Flüssigkeitsaufnahme messen sowie Abweichungen von Normwerten melden und zur autonomen Gebäudesteuerung, z. B. der Temperatur oder des Lichts sowie von Türen und Fenstern, eingesetzt werden (Daum 2017, S. 21 ff; vgl. Güttler et al. 2015, S. 268 ff; Bräutigam et al. 2017, S. 14; Klein 2011, S. 91; Vincent und Creteur 2017, S. 2). Personenortungssysteme können u. a. bei Menschen mit demenziellen Erkrankungen z. B. in der Geriatrie angewendet werden und eine Pflegekraft benachrichtigen, wenn eine Person einen festgelegten Bereich verlässt (Daum 2017, S. 30; Vincent und Creteur 2017, S. 3). Die entsprechenden Daten können zusätzlich an ein mobiles Endgerät gesendet werden (Bräutigam et al. 2017, S. 14; Vincent und Creteur 2017, S. 2).

Es fehlt allerdings häufig die umfassende Verknüpfung und Analyse der Informationen aus den einzelnen Bereichen, da viele dieser Geräte noch nicht mit elektronischen Dokumentationssystemen vernetzt sind (Daum 2017, S. 29; Hielscher 2014, S. $27 \mathrm{f}$.). So ist die Überwachung von Vitalparametern auf Intensivstationen zwar weit verbreitet, allerdings bleibt die Erfassung und Auswertung der Daten auf diese Station beschränkt. Die mithilfe von Telemonitoring-Geräten gesammelten Daten können basierend auf Algorithmen ausgewertet werden und eine bestimmte Behandlungsoption nahelegen. Die Geräte können aber auch eigenständig auf Veränderungen reagieren (vgl. Wong et al. 2015, S. 945; vgl. Vincent und Creteur 2017, S. 2; Daum 2017, S. 25; Mildner et al. 2017, S. 18) wie z. B. Beatmungsgeräte, die die Atmung des Patienten kontinuierlich überwachen, dessen Zustand diagnostizieren und basierend auf der Diagnose verschiedener Lungenbedingungen und Zielwerte den Beatmungsprozess automatisch anpassen (Mildner et al. 2017, S. 19).

Ein weiteres Feld assistierender Technik ist mit dem Begriff Augmented Reality charakterisiert (Neumuth 2016). Anwendung finden können derartige realitätserweiternden Technologien möglicherweise, um z. B. mithilfe von Datenbrillen virtuelle Informationen und Objekte in das Sichtfeld des Nutzers einzublenden. Die Datenbrille kann z. B. Informationen aus der elektronischen Pflegedokumentation, Anleitungen in Form von Fotos und Videos sowie Vitalparameter zeitgleich mit dem Pflegeprozess anzeigen (Daum 2017, S. 22; Neumuth 2016, S. 1; Mildner et al. 2017, S. 23). Dementsprechend kann auch in Echtzeit die Präsenz von räumlich distanzierten Personen auf die Datenbrille projiziert werden, um deren Expertise oder Handlungsanweisungen zu nutzen. Ferner wird die Anwendung von Augmented Reality in der Aus- und Weiterbildung diskutiert, um an realitätsnahen Fällen praxisorientiert lernen zu können (Neumuth 2016, S. 2). 
Technische Assistenzsysteme können zudem zur Unterstützung bei Tätigkeiten, die körperlich belastend sind oder nicht unmittelbar die Pflege am Menschen betreffen, eingesetzt werden. So können am Körper getragene Exoskelette die Pflegekräfte beim Heben und Tragen, z. B. beim Transfer von Patienten oder deren Umlagerung, entlasten und somit berufsbedingten Muskel-Skelett-Erkrankungen vorbeugen (Merda et al. 2018, S. 24; Daum 2017, S. 28).

Ein anderer Einsatzbereich mit unterstützenden Wirkungen durch assistierende Techniken stellt die Krankenhauslogistik dar (Kriegel 2012). So ist es in der Wäsche-, Arznei- und Medikalproduktelogistik möglich, die Pflegekräfte durch Assistenzsysteme zu entlasten. Als Beispiele können der intelligente Pflegewagen (Graf et al. 2018) sowie digitale Schranksysteme genannt werden (Buck et al. 2015). Pflegewagen können auf Abruf oder automatisch zu den Pflegekräften fahren, Pflegeutensilien bereitstellen sowie den Verbrauch dieser dokumentieren und zudem über mobile Endgeräte Zugang zur elektronischen Pflegedokumentation bieten (Graf et al. 2018; Daum 2017, S. 24). Derartige Systeme tragen prinzipiell nicht nur zu einer Entlastung und effizienteren Versorgung bei, sondern auch zur Qualitätssicherung.

\subsubsection{Fazit}

Abschließend bleibt festzuhalten, dass zurzeit zwar relativ wenige der aufgeführten Technologien in deutschen Krankenhäusern verbreitet sind, allerdings dürften Informations- und Kommunikationstechnologien, Roboter und technische Assistenzsysteme in den nächsten Jahren vermehrt verwendet bzw. eingesetzt werden. Ursächlich hierfür dürfte u. a. sein, dass sich die Kosten-Nutzen-Relationen dieser Techniken verbessern werden und die derzeit noch vorwiegend in Projekten verwendeten Systeme Alltagstauglichkeit erreichen.

Es ist davon auszugehen, dass der Einsatz dieser assistierenden Technologien Auswirkungen auf die Arbeitsorganisation und Rolle der Pflegekräfte haben wird. Zudem erfordert der Einsatz zusätzliche Kenntnisse, die über die bisher mit der Pflege verbundenen hinausgehen, was Auswirkungen auf die Aus- und Weiterbildung der Pflegekräfte haben dürfte (Bendig et al. 2017, S. 4 f.). Unsicherheit besteht in der Literatur allerdings über das Ausmaß der Veränderungen, wie im nächsten Abschnitt erläutert wird.

\subsection{Auswirkungen auf die Arbeits- organisation und die Rolle der Pflegekräfte}

Die digitalen Technologien können sich prinzipiell in mehrfacher Weise auf die Pflege im Krankenhaus auswirken. So ergeben sich einerseits Auswirkungen auf die Strukturen und Prozesse im Krankenhaus und andererseits auf die Rollen und das Selbstverständnis der Pflegekräfte.

\subsubsection{Einfluss auf die Strukturen und Prozesse der Pflege}

Die Umstellung von der handschriftlichen auf die elektronische Dokumentation, z. B. durch die Eingabe mittels mobiler Endgeräte und damit einhergehend durch die Verwendung von Textbausteinen, kann prinzipiell zu zeitlichen Entlastungen bei der Dokumentation beitragen (Rösler et al. 2018, S. 24; Daum 2017, S. 37). Eine weitere Zeitersparnis wäre möglich, wenn Daten z. B. durch Monitoringsysteme automatisch erfasst und in das System übertragen würden (Höhmann und Schwarz 2017, S. 158; Daum 2017, S. 37). Die Effizienzsteigerung aufgrund der Zeitersparnis bei der Dokumentation kann genutzt werden, um mehr Zeit für die Bedarfe des zu Pflegenden aufwenden zu können oder auch den personalen Austausch zu fördern (Meißner 2017, S. 160 f.; Daum 2017, S. 15; Vincent und Creteur 2017, S. 2; vgl. Orians und Reisach 2017, S. 49; Hielscher 2014, S. 35). Durch die digitale Erfassung kann zudem eine Qualitätssteigerung in der pflegerischen Versorgung erreicht werden, da sie dazu beitragen kann, die Reliabilität sowie Validität und damit die Belastbarkeit der verfügbaren Informationen zu verbessern.

Der Austausch zwischen den am Versorgungsprozess beteiligten Akteuren durch eine besser zugängliche, transparentere und standardisierte 
elektronische Dokumentation kann zur besseren Koordination des Versorgungsprozesses führen und somit auch die Qualität der Pflege verbessern (Rösler et al. 2018, S. 24; Bräutigam et al. 2017, S. 41 f.; Roland Berger GmbH et al. 2017, S. 30). Allen am Versorgungsprozess Beteiligten können die gleichen Informationen zur Verfügung stehen, sodass diese eine bessere Vorstellung vom Pflegeund Gesundheitszustand des Patienten erhalten (Rösler et al. 2018, S. 24). Die beteiligten Ärzte, Therapeuten sowie das Pflegepersonal hätten dann einen direkten, stations- und einrichtungsunabhängigen Zugriff auf die Patientendaten und müssten sich diese nicht erst bei Bedarf beschaffen oder ein weiteres Mal erheben (Rösler et al. 2018, S. 24; Roland Berger GmbH et al. 2017, S. 45; Hößl 2013, S. 25; vgl. Ploch und Werkmeister 2017, S. 33).

Auch kann durch die elektronische Dokumentation, die sich an Pflegeprozessen nach Expertenstandards und an pflegewissenschaftlichen Modellen orientiert, das evidenzbasierte Handeln der Pflegekräfte verbessert und auch hierdurch die Qualität der Pflege erhöht werden (Rösler et al. 2018, S. 24; Hößl 2013, S. 25; vgl. Sowinski et al. 2013, S. 53). So können den Pflegekräften beispielsweise mithilfe der elektronischen Dokumentation Vorschläge zu anliegenden Prozessschritten unterbreitet und somit die Entscheidungsfindung, wie der Patient versorgt werden soll, erleichtert werden und evidenzbasiert erfolgen (Hößl 2013, S. 25). Die Nutzung standardisierter Fachbegriffe kann zudem die Nachvollziehbarkeit und Verständlichkeit der Patientendaten auch für Fachkräfte aus anderen Einrichtungen oder Sektoren erhöhen (Rösler et al. 2018, S. 24).

Aus der elektronischen Dokumentation kann auch der Personal- und Zeitbedarf für den Pflegeprozess abgeleitet und zur effizienteren Arbeitsorganisation sowie zur Dienstplanung genutzt werden (Rösler et al. 2018, S. 25; Hielscher et al. 2015, S. 9 f.; Hößl 2013, S. 24 f.; vgl. Merda et al. 2018, S. 90). Gelingt es, die Arbeitsabläufe effizienter zu gestalten, können gegebenenfalls die Arbeitsintensität und Überstunden der Pflegekräfte reduziert werden. Nachteilig kann sich dieses System jedoch auf die Belastung der Pflegekräfte auswirken, wenn es ausschließlich zur Reduzierung der Lohnkosten genutzt werden würde, indem das Pflegepersonal verringert würde (vgl. Rösler et al. 2018, S. 25; vgl. Hielscher 2014, S. 35).

Der Einsatz von elektronischen Dokumentationssystemen kann durch die erhöhte Informationstransparenz allerdings auch zu mehr Kontrollmöglichkeiten aller und durch alle am Pflegeprozess Beteiligten führen. Nicht nur die Arbeitsprozesse, sondern auch das Verhalten, die Arbeitsleistung, Zeitaufwendungen für bestimmte Aufgaben und etwaige Fehler des Pflegepersonals können mithilfe derartiger Systeme abgebildet werden und somit zu einem erhöhten Leistungsdruck für die Pflegekräfte führen (Hielscher und Kirchen-Peters 2017, S. 29; Hielscher et al. 2015, S. 12; Hielscher 2014, S. 35; vgl. Ploch und Werkmeister 2017, S. 48; Bräutigam et al. 2017, S. 43 f.). So können auch Systeme zur Anwendung kommen, die Zeitspannen für die Bewältigung bestimmter Aufgaben vorgeben und beispielsweise mit akustischen Signalen den Ablauf der Zeit ankündigen. Derartige Systeme können zu erhöhtem Stress sowie Störungen der Arbeit und der Pflegekraft-Patienten-Interaktion führen und die Qualität der Pflege negativ beeinflussen (Hielscher et al. 2016, S. 11; vgl. Bräutigam et al. 2017, S. 43 f.). Eine Aufzeichnung aller Bewegungen der Pflegekraft z. B. mithilfe intelligenter Berufskleidung kann zudem Persönlichkeitsrechte verletzen und Fragen des Datenschutzes aufwerfen (Ploch und Werkmeister 2017, S. 22; vgl. Evans et al. 2018, S. 5).

Es wird kontrovers diskutiert, ob sich tatsächlich Zeitgewinne einstellen, wenn ja und wofür diese Zeit dann alternativ aufgewendet wird (Hielscher und Kirchen-Peters 2017, S. 29). Zum einen kann es sein, dass durch die potenziellen Zeiteinsparungen, durch Berücksichtigung von Expertenstandards oder weitere Funktionen der Dokumentationssysteme mehr dokumentiert wird, sich der Dokumentationsaufwand also erhöht (Rösler et al. 2018, S. 24; Ploch und Werkmeister 2017, S. 34). Von einem höheren Zeitaufwand ist auch auszugehen, wenn zusätzlich zur elektronischen Dokumentation weiter an der papierbasierten Dokumentation festgehalten und so Parallelstrukturen entstehen (Rösler et al. 2018, S. 25) oder wenn die Daten aus einem elektronischen System, z. B. dem Abrechnungssystem, nicht in das zur Pflegedokumentation verwendete integrierbar sind und somit redundant erfasst werden (Höhmann und Schwarz 2017, 
S. 159 f.; vgl. Merda et al. 2018, S. 83). Ferner ist zu beachten, dass in der Einführungs- bzw. Umstellungsphase der papierbasierten auf die elektronische Dokumentation mehr Zeit für das Erlernen der Nutzung der Technik und gegebenenfalls für die Anpassung der routinierten Arbeitsabläufe an die neuen Strukturen aufgebracht werden muss (vgl. Ploch und Werkmeister 2017, S. 34). Ob der gewonnene Zeitaufwand wirklich für pflegenahe Tätigkeiten und nicht andere Aufgaben verwendet wird, wird kritisch hinterfragt (Ploch und Werkmeister 2017, S. 26).

Außerdem ist zu fragen, ob nicht die standardisierte Dokumentation - auch unter Anbetracht des vorherrschenden Zeitdrucks auf vielen Stationen dazu führt, dass (subjektive) Eindrücke, die sich nicht in der Dokumentationsmaske abbilden lassen, wie z. B. Gerüche oder die seelische Verfassung des Patienten, nicht mehr erhoben würden (Rösler et al. 2018, S. 25; vgl. Hielscher 2014, S. 35). Zudem kann es sein, dass sich die Pflegekräfte an die starren Dokumentationsvorgaben, die durch den Pflegeprozess führen, halten und danach handeln, sodass ihr eigenständiges Denken und (situatives) Handeln vernachlässigt wird (Hielscher und Kirchen-Peters 2017, S. 29 f.; Sowinski et al. 2013, S. 53). Wenn die elektronische Dokumentation den persönlichen Austausch zwischen Pflegekräften ersetzen würde, kann es zudem sein, dass wichtige Informationen, die z. B. auf Intuition oder langjährigen Berufserfahrungen beruhen, nicht mehr dokumentiert und damit nicht weitergegeben würden (Rösler et al. 2018, S. 25; Hielscher et al. 2015, S. 10 f.). Kann die Dokumentation den Pflegeprozess nur unzureichend vorgeben, da z. B. kontextbedingt alternative patientenindividuelle Pflegemaßnahmen nötig sind, wird somit möglicherweise die Qualität der Pflege beeinträchtigt (Hielscher et al. 2015, S. 12 f.; vgl. Hülsken-Giesler und Krings 2015, S. 7; vgl. Sowinski et al. 2013, S. 53; vgl. Remmers 2015, S. 14 f.).

Während mehrere Studien eine gestiegene Qualität durch die präzisere elektronische Dokumentation belegen können, gibt es widersprüchliche Studienergebnisse zur Zeiteinsparung, die durch die elektronische Dokumentation erreicht wird (Hielscher et al. 2015, S. 10 f.; Ploch und Werkmeister 2017, S. 48). Generell bestehen zu den Auswirkungen der elektronischen Dokumentation auf das
Handeln der Pflegekräfte noch Forschungsbedarf (Hielscher et al. 2015, S. 13; Höhmann und Schwarz 2017, S. 154).

Telemedizinische Technologien, die eine videobasierte Kommunikation mit Fachkräften zulassen, können zusätzlich den Austausch zwischen den Professionen erleichtern. Zeiteinsparungen und Arbeitserleichterungen können sich dadurch ergeben, dass fachliche oder auch dolmetschende Expertise schneller und ortsunabhängig eingeholt werden kann (Rösler et al. 2018, S. 45; Merda et al. 2018, S. 93). Auch die pflegerische Expertise kann aus der Ferne, gegebenenfalls von zu Hause aus, erbracht werden und möglicherweise eine bessere Vereinbarkeit von Beruf und Privatleben gewähren und so die Mitarbeiterzufriedenheit erhöhen (Merda et al. 2018, S. 93). Hinsichtlich des Einsatzes dieser Technologien ist allerdings zu beachten, dass der fehlende persönliche Kontakt zum Patienten wiederum die Pflegequalität negativ beeinflussen kann (Merda et al. 2018, S. 84; Rösler et al. 2018, S. 46; Hielscher et al. 2015, S. 15).

Service-Roboter können potenziell pflegeferne, zeitaufwendige und körperlich anstrengende Routinetätigkeiten übernehmen, ihr Einsatz kann somit zu Zeitgewinnen führen und die Pflegekräfte körperlich entlasten (Graf et al. 2013, S. 1146; Ploch und Werkmeister 2017, S. 23; Merda et al. 2018, S. 99; Klein und Cook 2009, S. 24). Auch Kontrollund Überwachungsaufgaben können von derartigen Robotern übernommen werden, die dann nicht mehr oder nur noch teilweise von Pflegekräften durchgeführt werden müssten und somit zusätzliche Zeitersparnisse mit sich bringen können (Ploch und Werkmeister 2017, S. 31 f.). Die gewonnene Zeit kann dann für die pflegenahe, komplexe Beziehungsarbeit oder auch präventive sowie rehabilitative Behandlungen eingesetzt werden, die nicht von Robotern übernommen werden können (Merda et al. 2018, S. 99; vgl. Rösler et al. 2018, S. 56). Da bisher nur wenige Service-Roboter in deutschen Krankenhäusern eingesetzt werden, besteht noch Forschungsbedarf hinsichtlich der Abschätzung der Effekte dieser Technologien auf die Arbeitsweise der Pflegekräfte (Hielscher 2014, S. 33; vgl. Ploch und Werkmeister 2017, S. 26).

Gerade auf geriatrischen Stationen und hinsichtlich der Betreuung von Menschen mit demen- 
ziellen Erkrankungen können Assistenztechnologien potenziell die zeitaufwendige und psychischfordernde Betreuung von zu Pflegenden erleichtern (Merda et al. 2018, S. 96; vgl. Hielscher et al. 2015, S. 15). Wearables können eingesetzt werden, damit sich diese auf der Station frei bewegen können, aber trotzdem nicht durchgängig von einer Pflegekraft beobachtet werden müssten (Rösler et al. 2018, S. 35). Dies kann auch die Pflegekräfte psychisch entlasten, die befürchten, die Aufsichtspflicht zu verletzen, wenn sie die Pflegebedürftigen allein und unbeobachtet lassen müssen (Sowinski et al. 2013, S. 40; Merda et al. 2018, S. 85; Hielscher et al. 2015, S. 15). Weitere zeitliche sowie psychische Entlastungen können möglicherweise durch sozial-interaktiven Roboter erreicht werden, die mit den Patienten interagieren (vgl. Merda et al. 2018, S. 87). Hinsichtlich derartiger Technologien wird jedoch kritisiert, dass die Patienten und deren Angehörige diese Technologien ablehnen, wenn sie sich aufgrund des Einsatzes von Robotertieren oder -puppen nicht ernst genommen fühlen oder eine ständige Überwachung aus ethischen Gründen ablehnen und sich diese Effekte auch auf die Beziehungsarbeit auswirken (vgl. Merda et al. 2018, S. 99; vgl. Sowinski et al. 2013, S. 41). Auch für diese Technologien gilt, dass Studien fehlen, in denen die Effekte des Einsatzes dieser Technologien analysiert werden (Hielscher et al. 2015, S. 15; vgl. Merda et al. 2018, S. 100).

Weitere körperliche Entlastung können Exoskelette bringen, die durch Kraftübertragung körperlich anstrengende Pflegehandlungen, wie z. B. die Lagerung von den zu Pflegenden, erleichtern (Merda et al. 2018, S. 87; Rösler et al. 2018, S. 51). Dies kann auch dazu führen, dass Fehlhaltungen und berufsbedingte körperliche Schädigungen wie z. B. Bandscheibenvorfälle abnehmen und somit Krankheitstage oder ein vorzeitiger Ausstieg aus dem Beruf reduziert würden (Merda et al. 2018, S. 17 ff.; vgl. Rösler et al. 2018, S. 51).

\subsubsection{Einfluss auf das Berufsbild und Selbstverständnis der Pflegekräfte}

Hinsichtlich der Auswirkungen der Digitalisierung im Krankenhaus auf das Berufsbild der Pflegekräfte existieren zurzeit in der Literatur unterschiedliche Ansätze. Einerseits wird in der Literatur nicht von einer Substitution der Pflegekräfte durch assistierende Technologien ausgegangen. Gerade die komplexe, feinmotorische, auf einem hohen Grad von sozialer Intelligenz beruhende und erfahrungsbasierte Bezugspflege könne durch derartige Technologien nur bedingt ersetzt werden (Merda et al. 2018, S. 99; Rösler et al. 2018, S. 56; Orians und Reisach 2017, S. 37). Auch die Pflegekräfte selbst gehen nicht von einem substitutiven Prozess durch Digitalisierung aus. Vielmehr dürften sich komplementäre Beziehungen entwickeln und gegebenenfalls Tätigkeiten durch die Digitalisierung hinzukommen (Bräutigam et al. 2017, S. 38 ff.).

Andererseits wird eine Deprofessionalisierung der Pflege befürchtet, wenn durch Technikeinsatz die auf Berührung und körperliche Präsenz der Pflegekraft beruhende Bezugspflege reduziert würde und fachfremde Aufgaben übernommen werden müssten (Rösler et al. 2018, S. 46; Meißner 2017, S. 164; Remmers 2015, S. 18; Hülsken-Giesler und Wiemann 2015, S. 50; Friesacher 2010, S. 302 f.). So kann es sein, dass den Fachkräften nur noch technische, administrative und überwachende Aufgaben zufallen, die distanziert vom zu Pflegenden erbracht werden und somit zusätzlich Wissen sowie Kompetenzen verloren gehen (Höhmann und Schwarz 2017, S. 154; Ploch und Werkmeister 2017, S. 48; Friesacher 2010, S. 302 f.; vgl. Bräutigam et al. 2017, S. 16 f.). Auch können potenziell Tätigkeiten der Pflegefachkräfte mithilfe neuer Technik von Pflegehilfskräften übernommen werden (Ploch und Werkmeister 2017, S. 39; Hielscher 2014, S. 11). Ferner wird von einer Deprofessionalisierung der Pflege und einem Verlust der Autonomie der Pflegekräfte gesprochen, wenn sich die pflegerischen Tätigkeiten an den Vorgaben bzw. Erfordernissen der Technik ausrichten und somit nicht mehr am Patienten orientieren würden (Bräutigam et al. 2017, S. 16 f.; Friesacher 2010, S. 303 f.).

Des Weiteren wird kontrovers diskutiert, inwieweit Digitalisierung die Attraktivität des Pflegeberufs erhöhen kann. Laut Remmers (2016, S. 202) ist nicht von einer derartigen Steigerung durch Technik auszugehen (vgl. auch Ploch und Werkmeister 2017, S. 39). Der Pflegeberuf kann jedoch aufgrund von Kompetenz- und Aufgabenerweiterungen 
durch die Digitalisierung attraktiver werden. Zum Beispiel können Pflegekräfte möglicherweise unter telemedizinischer (An-)Leitung des ärztlichen Personals teilweise die medizinische Versorgung und somit zusätzliche Aufgaben übernehmen (Hielscher 2014, S. 36). Derartige Tätigkeiten bedürfen jedoch gegebenenfalls weiterer Kompetenzen und somit auch Qualifikationen (Hielscher 2014, S. 36). Ferner kann der Erwerb von Technikkompetenzen zu einer höheren Anerkennung der Pflege gegenüber anderen Professionen führen, was ebenfalls die Attraktivität steigern kann (vgl. Evans et al. 2018, S. 5; vgl. Ploch und Werkmeister 2017, S. 39 f.).

Einigkeit besteht hingegen bei der Feststellung, dass im Zuge der Digitalisierung der Erwerb von Technikkompetenzen unerlässlich ist. So wird argumentiert, dass es nicht nur in der pflegerischen Ausbildung, sondern auch im Rahmen von regelmäßigen Fort- und Weiterbildungen wichtig ist, sich den Umgang mit neuester Technik anzueignen (Bendig et al. 2017; Hübner et al. 2017, S. 2; vgl. Evans et al. 2018, S. 9; Ploch und Werkmeister 2017, S. 49). In einer Empfehlung der Arbeitsgruppe "Informationsverarbeitung in der Pflege“ der Deutschen Gesellschaft für Medizinische Informatik, Biometrie und Epidemiologie (GMDS), der Österreichischen Gesellschaft für Pflegeinformatik (ÖGPI) und der Schweizerischen Interessensgruppe Pflegeinformatik (IGPI) innerhalb des Schweizer Berufsverbandes der Pflegefachfrauen und Pflegefachmänner (SBK) werden Kernkompetenzen vorgestellt, die in der Pflegeausbildung Berücksichtigung finden sollen (Hübner et al. 2017, S. 4): Neben dem Umgang u. a. mit der elektronischen Pflegedokumentation, der Qualitätssicherung und dem Prozessmanagement wird in der Empfehlung auch auf die mit der Technik zusammenhängenden rechtlichen sowie ethischen Kompetenzbereiche verwiesen (vgl. auch Hackl et al. 2016, S. 383). Die Gesellschaft für Informatik hebt in ihren Handlungsempfehlungen für die Entwicklung und den Erwerb digitaler Kompetenzen in Pflegeberufen zudem hervor, dass ein kritischer und selbstbestimmter Umgang mit digitaler Technik u. a. durch das Verstehen der Aus- und Wechselwirkungen derartiger Technologien wichtig ist (Bendig et al. 2017, S. 7 ff.). Ergänzend sei es wichtig, grundlegende Kompetenzen, die die Anpassung an sich verändernde bzw. neue Technologien vereinfachen, aber auch auf die einzelnen Sektoren und Pflegekontexte abgestimmte Technikkompetenzen zu vermitteln (Bendig et al. 2017, S. 9 ff.; Hackl et al. 2016, S. 386). Der Erwerb von Technikkompetenzen kann auch eine stärkere Einbindung von Pflegekräften und damit der pflegerischen Expertise in den Entwicklungsprozess neuer Technik ermöglichen (Hackl et al. 2016, S. 383; Bendig et al. 2017, S. 10; Rösler et al. 2018, S. 38). Auch wäre es durch die zusätzlichen Kompetenzen einfacher möglich, Pflegekräfte stärker in Entscheidungen über den Einsatz von Technik einzubeziehen (Fuchs-Frohnhofen et al. 2018; Bräutigam et al. 2017, S. 24; Evans et al. 2018, S. 5; Merda et al. 2018, S. 144; Rösler et al. 2018, S. 38; Bendig et al. 2017, S. 15; Hackl et al. 2016, S. 383).

Ein abgestimmtes und einheitliches Kompetenzprofil, die Einbindung der Technikausbildung in bestehende Curricula der Pflegeausbildung und flächendeckende Fort- und Weiterbildungsmaßnahmen fehlen jedoch bis dato zum Großteil (Hackl et al. 2016, S. 383; Bendig et al. 2017, S. 10 ff.; Evans et al. 2018, S. 9; vgl. Bräutigam et al. 2017, S. 47; Roland Berger GmbH et al. 2017, S. 35; Merda et al. 2018, S. 147 f.).

\subsection{Fazit und Ausblick}

Es zeigt sich, dass digitale Technologien in der Krankenhauspflege zur Informationsverarbeitung, Dokumentation und Organisation sowie Kommunikation zwischen den Pflegekräften ein hohes Potenzial aufweisen und in vielfältigen Zusammenhängen eingesetzt werden können. Da eine qualitativ hochwertige Pflege auf die individuelle Lebenssituation und die persönlichen Bedürfnisse des Pflegebedürftigen ausgerichtet und somit auf komplexe und mitunter situative Entscheidungen der Pflegekräfte angewiesen ist, werden derartige Technologien voraussichtlich nicht zu einer Substitution der Pflegefachkräfte führen (Merda et al. 2018, S. 99; Rösler et al. 2018, S. 56; Orians und Reisach 2017, S. 37; Hielscher et al. 2016, S. 9). Vielmehr sind durch die assistierenden Systeme potenziell Effizienz-, Effektivitäts- und Qualitätssteigerungen möglich. So können Synergieeffekte durch Informa- 
tionstransparenz sowie Veränderungen der Arbeitsprozesse und -organisation zu effizienteren Arbeitsabläufen für die Pflegekräfte führen. Allerdings fehlen hierzu noch entsprechende Analysen. In Einzelstudien gibt es zwar Hinweise, aber repräsentative Studien mit belastbaren Ergebnissen liegen nicht vor und standardisierte Verfahren im Rahmen von Health Technology Assessment (HTA) fehlen weitgehend (Mähs 2017, S. 125; Meißner 2017, S. 160 f.; vgl. Merda et al. 2018, S. 146). Für die Finanzierung über die Regelversorgung sind entsprechende Kosten-Nutzen-Analysen erforderlich (Fachinger 2018, S. 63; Fachinger et al. 2015, S. 63 f.; Ploch und Werkmeister 2017, S. 32).

Durch eine zunehmende Digitalisierung hin zu einer integrierten, flächendeckenden und systematischen Nutzung digitaler Technologien ergeben sich zudem Veränderungen im Hinblick auf die Aufgaben, die Rolle und auch das Selbstverständnis der Pflegekräfte. Dementsprechend ist es relevant, die Auswirkungen dieser Technologien auf die Arbeitsorganisation und -bedingungen sowie das Berufsbild und Selbstverständnis der Pflege und auch der Pflegebedürftigen aufzuzeigen (Fachinger et al. 2017, S. 34; Fuchs-Frohnhofen et al. 2018, S. 9 f.; Rösler et al. 2018, S. 56; Merda et al. 2018, S. 143 f.; Bräutigam et al. 2017, S. 15; Ploch und Werkmeister 2017, S. 34; Höhmann und Schwarz 2017, S. 152; Hielscher et al. 2015, S. 16).

Insgesamt gesehen kann durch die höhere Verbreitung und Nutzung digitaler Technologien in Krankenhäusern den zukünftigen Herausforderungen, zu denen insbesondere die Zunahme an Pflegebedürftigen gezählt wird, begegnet werden. Allerdings müssen dazu bestimmte Anforderungen erfüllt werden, zu denen $u$. a. technisch-strukturelle Voraussetzungen wie die Umsetzung von Standards und Normen zur Verbesserung der Interoperabilität und der Ausbau von leistungsfähigen und sicheren Breitbandverbindungen, konstante bzw. verlässliche gesetzliche und institutionelle Rahmenbedingungen sowie die Aus- und Weiterbildung der Pflegekräfte in bestehenden und neuen Berufsfeldern zu zählen sind (Fachinger 2018, S. 53; Merda et al. 2018, S. 91; Roland Berger GmbH et al. 2017, S. 28; Fachinger et al. 2015, S. 63 f.).

\section{- Danksagung}

Wir bedanken uns bei Frau Sollmann und den unbekannten Reviewern für ihre hilfreichen Anmerkungen und Vorschläge.

\section{Literatur}

Apt W, Bovenschulte M, Hartmann EA, Wischmann S (2016) Foresight-Studie „Digitale Arbeitswelt", Forschungsbericht, Bundesministerium für Arbeit und Soziales, Berlin

Augurzky B, Krolop S, Mensen A, Pilny A, Schmidt CM, Wuckel C (2018) Krankenhaus Rating Report 2018. Personal Krankenhäuser zwischen Wunsch und Wirklichkeit. Krankenhaus Rating Report, medhochzwei, Heidelberg

Ausserhofer D, Zander B, Busse R, Schubert M, De Geest S, Rafferty AM, Ball J, Scott A, Kinnunen J, Heinen M, Strømseng Sjetne I, Moreno-Casbas T, Kózka M, Lindqvist R, Diomidous M, Bruyneel L, Sermeus W, Aiken LH, Schwendimann R (2014) Prevalence, patterns and predictors of nursing care left undone in European hospitals: results from the multicountry cross-sectional RN4CAST study. BMJ Quality \& Safety 23(2):126-135

Ball MJ, Hannah KJ, DuLong D, Newbold SK, Sensmeier JE, Skiba DJ, Troseth MR, Gugerty B, Hinton Walker P, Douglas JV (Hrsg) (2011) Nursing Informatics. Where Technology and Caring Meet (Health Informatics). Springer, London

Bendig T, Bleses P, Breuer J, Buhr R, Egbert N, Hübner UH, Koubek J, Krupka D, Lutze M, Müller L-S, Regitz C, Rutha M, Schnellhammer M, Schubert M, Schwarze B, Weißbach R, Welskop-Deffaa EM (2017) Leitlinien Pflege 4.0: Handlungsempfehlungen für die Entwicklung und den Erwerb von digitalen Kompetenzen in Pflegeberufen des Beirats IT-Weiterbildung der Gesellschaft für Informatik e. V. in Zusammenarbeit mit Partnerinnen und Partnern aus Pflegepraxis, Verbänden und Wissenschaft. Gesellschaft für Informatik e. V., Berlin

van den Berg N, Schmidt S, Stentzel U, Mühlan H, Hoffmann W (2015) Telemedizinische Versorgungskonzepte in der regionalen Versorgung ländlicher Gebiete. Bundesgesundheitsblatt - Gesundheitsforschung - Gesundheitsschutz 58(4):367-373

Bräutigam C, Enste P, Evans M, Hilbert J, Merkel S, Öz F (2017) Digitalisierung im Krankenhaus. Mehr Technik - bessere Arbeit? Study Vol. 364. Hans-Böckler-Stiftung, Düsseldorf Brüggemann J, Coners E, Franzen-Krapoth $\mathrm{H}$, Hollenbach D, Kowalski I, Mittnacht B, Muck T, Vogt K, Wenzel D (2017) Qualität in der ambulanten und stationären Pflege. 5. Pflege-Qualitätsbericht des MDS nach § 114a Abs. 6 SGB XI. MDS-Pflege-Qualitätsberichte, Medizinischer Dienst des Spitzenverbandes Bund der Krankenkassen e. V. (MDS), Essen

Bruyneel L, Li B, Ausserhofer D, Lesaffre E, Dumitrescu I, Smith HL, Sloane DM, Aiken LH, Sermeus W (2015) Organization of Hospital Nursing, Provision of Nursing Care, 
and Patient Experiences With Care in Europe. Medical Care Research and Review 72(6):643-664

Buck M, Schröder J, Woratschek H, Tomanek DP, Stadtelmann

M, Horbel C, Weismann F (2015) Benchmarking der Schrankfachversorgung: Ergebnisse einer Studie mit Kliniken und Dienstleistern. In: Woratschek H, Schröder J, Eymann T, Buck M (Hrsg) Wertschöpfungsorientiertes Benchmarking: Logistische Prozesse in Gesundheitswesen und Industrie. Springer, Berlin Heidelberg, S 183-211

Bundesministerium für Arbeit und Soziales (2017) Weissbuch Arbeiten 4.0, Arbeit Weiter Denken, Bundesministerium für Arbeit und Soziales, Abteilung Grundsatzfragen des Sozialstaats, der Arbeitswelt und der sozialen Marktwirtschaft, Berlin

Bundesregierung (2018) Antwort der Bundesregierung auf die Kleine Anfrage der Abgeordneten Kordula Schulz-Asche, Maria Klein-Schmeink, Dr. Kirsten Kappert-Gonther, weiterer Abgeordneter und der Fraktion BÜNDNIS 90/DIE GRÜNEN - Drucksache 19/1550 -. Unbesetzte Stellen in der Alten- und Krankenpflege. Bundestags-Drucksache 19/1803, Deutscher Bundestag, Berlin

Bundesverband Pflegemanagement (Hrsg) (2017) Digitale Dokumentation in der Pflege. Ein Praxis-Handbuch. Bundesverband Pflegemanagement, Berlin

Dahl TS, Boulos MNK (2013) Robots in Health and Social Care: A Complementary Technology to Home Care and Telehealthcare? Robotics 3(1):1-21

Dall'Ora C, Griffiths P, Ball J, Simon M, Aiken LH (2015) Association of $12 \mathrm{~h}$ shifts and nurses' job satisfaction, burnout and intention to leave: findings from a cross-sectional study of 12 European countries. BMJ Open 5(9). Open Access

Daum M (2017) Digitalisierung und Technisierung der Pflege in Deutschland. Aktuelle Trends und ihre Folgewirkungen auf Arbeitsorganisation, Beschäftigung und Qualifizierung. DAA-Stiftung Bildung und Beruf, Hamburg

Deutsche Stiftung Patientenschutz (2018) Stellungnahme zur Anhörung „Pflegepersonalmangel in den Krankenhäusern und in der Altenpflege" des Ausschusses für Gesundheit des Deutschen Bundestages am 18. April 2018 zu den Bundestagsdrucksachen 19/30, 19/79, 19/446 und 19/447. Patientenschutz Info-Dienst, Dortmund

Dörries M, Gensorowsky D, Greiner W (2017) Digitalisierung im Gesundheitswesen - hochwertige und effizientere Versorgung. Wirtschaftsdienst 97(10):692-696

Engelmann U, Schwind F (2017) Bildkommunikation in der Medizin: Vom PACS zum flächendeckenden E-HealthSystem. In: Müller-Mielitz S, Lux T (Hrsg) E-Health-Ökonomie. Springer, Wiesbaden, S 683-705

Evans M, Hielscher V, Voss D (2018) Damit Arbeit 4.0 in der Pflege ankommt. Wie Technik die Pflege stärken kann, Policy-Brief der Hans-Böckler-Stiftung, Düsseldorf

Fachinger U (2018) Altern und Technik: Anmerkungen zu den ökonomischen Potenzialen. In: Künemund H, Fachinger U (Hrsg) Alter und Technik. Sozialwissenschaftliche Befunde. Vechtaer Beiträge zur Gerontologie. Springer VS, Wiesbaden, S 51-68
Fachinger U, Nellissen G, Siltmann S (2015) Neue Umsatzpotenziale für altersgerechte Assistenzsysteme? Ausweitung der Regelversorgung im SGB V. Zeitschrift für Sozialreform 61(1):43-71

Fachinger U, Mähs M, Nobis S (2017) Selbstmanagement von informeller Pflege durch e-Health. Recht und Politik im Gesundheitswesen (RPG) 23(1):32-35

Frick Y, Baumberger D (2018) Sekundärnutzen von Pflegedaten für ökonomische Nachhaltigkeit durch automatisierte Ermittlung hochaufwändiger Pflegefälle aus der Patientendokumentation. In: Pfannstiel MA, Krammer S, Swoboda W (Hrsg) Digitale Transformation von Dienstleistungen im Gesundheitswesen IV: Impulse für die Pflegeorganisation. Springer, Wiesbaden, S 69-82

Friesacher H (2010) Pflege und Technik - eine kritische Analyse. Pflege \& Gesellschaft 15(4):293-313

Fuchs-Frohnhofen P, Blume A, Ciesinger K-G, Gessenich H, Hülsken-Giesler M, Isfort M, Jungtäubl M, Kocks A, Patz M, Weihrich M (2018) Memorandum „Arbeit und Technik 4.0 in der professionellen Pflege". MA\&T Sell \& Partner, Würselen

Grabbe Y, Nolting H-D, Loos S (2005) DAK-BGW Gesundheitsreport 2005 - Stationäre Krankenpflege. Arbeitsbedingungen und Gesundheit von Pflegenden in Einrichtungen der stationären Krankenpflege in Deutschland vor dem Hintergrund eines sich wandelnden Gesundheitssystems. Deutsche Angestellten-Krankenkasse (DAK), Berufsgenossenschaft für Gesundheitsdienst und Wohlfahrtspflege (BGW), Hamburg

Graf B, Heyer T, Klein B, Wallhoff F (2013) Servicerobotik für den demografischen Wandel. Mögliche Einsatzfelder und aktueller Entwicklungsstand. Bundesgesundheitsblatt - Gesundheitsforschung - Gesundheitsschutz 56(9):1145-1152

Graf B, King RS, Rößner A, Schiller C, Ganz W, Bläsing D, Fischbach J, Warner N, Bornewasser M (2018) Entwicklung eines intelligenten Pflegewagens zur Unterstützung des Personals stationärer Pflegeeinrichtungen. In: Pfannstiel MA, Krammer S, Swoboda W (Hrsg) Digitale Transformation von Dienstleistungen im Gesundheitswesen IV: Impulse für die Pflegeorganisation. Springer, Wiesbaden, S 25-49

Griffiths P, Dall'Ora C, Simon M, Ball J, Lindqvist R, Rafferty A-M, Schoonhoven L, Tishelman C, Aiken LH (2014) Nurses' Shift Length and Overtime Working in 12 European Countries: The Association With Perceived Quality of Care and Patient Safety. Medical Care 52(11):975-981

Güttler J, Georgoulas C, Linner T, Bock T (2015) Towards a future robotic home environment: a survey. Gerontology 61:268-280

Haase P (2018) Wertebeitrag von einrichtungsübergreifenden elektronischen Patientenakten. In: Hübner U, Esdar M, Hüsers J, Liebe J-D, Rauch J, Thye J, et al. (Hrsg) IT-Report Gesundheitswesen. Schwerpunkt - Wie reif ist die IT in deutschen Krankenhäusern? Befragung der bundesdeutschen Krankenhäuser. IT-Report Gesundheitswesen. Forschungsgruppe Informatik im Gesundheitswesen (IGW), Hochschule Osnabrück, Fakultät für Wirtschaftsund Sozialwissenschaften, S 15-19 
Hackl WO, Ammenwerth E, Ranegger R (2016) Bedarf an Fort- und Weiterbildung in Pflegeinformatik. Ergebnisse einer Umfrage. Pflegewissenschaft 18(7/8):381-387

Hämel K, Schaeffer D (2013) Who cares? Fachkräftemangel in der Pflege. Zeitschrift für Sozialrefom 59(4):413-431

Hielscher V (2014) Technikeinsatz und Arbeit in der Altenpflege. Ergebnisse einer internationalen Literaturrecherche, iso-Report (Vol. 1) Institut für Sozialforschung und Sozialwirtschaft (iso) e. V., Saarbrücken

Hielscher V, Kirchen-Peters S (2017) EDV-gestützte Dokumentation - wie wandelt sich die Pflegearbeit? QM-Praxis in der Pflege(4):28-30

Hielscher V, Kirchen-Peters S, Sowinski C (2015) Technologisierung der Pflegearbeit? Wissenschaftlicher Diskurs und Praxisentwicklungen in der stationären und ambulanten Langzeitpflege. Pflege \& Gesellschaft 20(1):5-19

Hielscher V, Nock L, Kirchen-Peters S (2016) Technikvermittlung als Anforderung in der Dienstleistungsinteraktion. Empirische Befunde zum Technikeinsatz in der Altenpflege. Zeitschrift Arbeit 25(1-2):3-19

Hien W (2017) „Man geht mit einem schlechten Gewissen nach Hause" - Krankenhausarbeit unter Ökonomisierungsdruck. Widersprüche 145(37):71-81

Höhmann U, Schwarz L (2017) Kompetenzanforderungen an pflegerische Führungskräfte in technikbezogenen Innovationsprozessen. In: Pfannstiel MA, Krammer S, Swoboda W (Hrsg), Digitale Transformation von Dienstleistungen im Gesundheitswesen III. Impulse für das Management. Springer Fachmedien, Wiesbaden, S 151-172

HößI I (2013) Sektorenübergreifende Kommunikation. Wie viel IT braucht die Pflege? Heilberufe / Das Pflegemagazin 65(5):24-26

Hübner U, Liebe J-D, Hüsers J, Thye J, Egbert N, HackI W, Ammenwerth E (2015) IT-Report Gesundheitswesen. Schwerpunkt Pflege im Informationszeitalter. IT-Report Gesundheitswesen. Forschungsgruppe Informatik im Gesundheitswesen (IGW) - Hochschule Osnabrück

Hübner U, Egbert N, Hackl W, Lysser M, Schulte G, Thye J, Ammenwerth E (2017) Welche Kernkompetenzen in Pflegeinformatik benötigen Angehörige von Pflegeberufen in den D-A-CH-Ländern? Eine Empfehlung der GMDS, der ÖGPI und der IGPI. GMS Medizinische Informatik, Biometrie und Epidemiologie 13(1)

Hübner U, Esdar M, Hüsers J, Liebe J-D, Rauch J, Thye J, Weiß J-P (2018) IT-Report Gesundheitswesen. Schwerpunkt Wie reif ist die IT in deutschen Krankenhäusern? Befragung der bundesdeutschen Krankenhäuser. IT-Report Gesundheitswesen. Forschungsgruppe Informatik im Gesundheitswesen (IGW) - Hochschule Osnabrück

Hülsken-Giesler M, Krings B-J (2015) Technik und Pflege in einer Gesellschaft des langen Lebens. Einführung in den Schwerpunkt. Technikfolgenabschätzung. Theorie und Praxis 24(2):4-11

Hülsken-Giesler M, Wiemann B (2015) Die Zukunft der Pflege - 2053: Ergebnisse eines Szenarioworkshops. Technikfolgenabschätzung. Theorie und Praxis 24(2):46-57
Klein B (2011) Technisierte Versorgung oder mehr Zeit für Kernaufgaben? Auswirkungen neuer Technologien auf die Pflegekräfte. Archiv für Wissenschaft und Praxis der sozialen Arbeit 42(3):86-98

Klein B, Cook G (2009) Robotik in der Pflege - Entwicklungstendenzen und Potenziale. Public Health Forum 17:23-24

Koenig S (2015) Freie Kapazitäten durch optimierte Versorgungslogistik. In: Woratschek H, Schröder J, Eymann T, Buck M (Hrsg) Wertschöpfungsorientiertes Benchmarking: Logistische Prozesse in Gesundheitswesen und Industrie. Springer, Berlin, Heidelberg, S 151-172

Kriegel J (2012) Krankenhauslogistik. Innovative Strategien für die Ressourcenbereitstellung und Prozessoptimierung im Krankenhauswesen. Springer Gabler, Wiesbaden

Leineweber C, Westerlund H, Chungkham HS, Lindqvist R, Runesdotter S, Tishelman C (2014) Nurses' Practice Environment and Work-Family Conflict in Relation to Burn Out: A Multilevel Modelling Approach. PLoS ONE 9(5): e96991

Madsack B, Walz M (2014) Abnahme und Konstanzprüfung an Bildwiedergabesystemen - was ändert sich mit der neuen DIN V 6868-157? Radiopraxis 7:195-210

Mähs M (2017) Evaluation of AAL-Technologies: Status Quo and Perspectives. Global Business \& Economics Anthology 2:124-132

Meißner A (2017) Technisierung der professionellen Pflege. Einfluss. Wirkung. Veränderung. In: Hagemann T (Hrsg), Gestaltung des Sozial- und Gesundheitswesens im Zeitalter von Digitalisierung und technischer Assistenz. Nomos, Baden-Baden, S 155-171

Merda M, Schmidt K, Kähler B (2018) Pflege 4.0 - Einsatz moderner Technologien aus der Sicht professionell Pflegender. Forschungsbericht. Berufsgenossenschaft für Gesundheitsdienst und Wohlfahrtspflege, Hamburg

Mildner R, Meyer J-U, Eckardt N, Hartung L, Kahlisch J, Biouchagiar J, Mildner M (2017) Krankenhaus 4.0, UniTransferKlinik Lübeck

Mischak R (2017) Wearables als Herausforderung im Gesundheitswesen - Revolutionieren Wearables das Gesundheitswesen im 21. Jahrhundert? In: Pfannstiel MA, Da-Cruz P, Mehlich H (Hrsg), Digitale Transformation von Dienstleistungen im Gesundheitswesen I: Impulse für die Versorgung. Springer, Wiesbaden, S 277-288

Müller B (2009) Betriebliches Gesundheitsmanagement im System Krankenhaus - Bestandsaufnahme und Ausblick. Expertise. Hans-Böckler-Stiftung, Düsseldorf

Neumuth T (2016) Augmented Reality in der Medizintechnik, VDE MedTech Expertenbeiträge. Verband der Elektrotechnik Elektronik Informationstechnik e. V., Frankfurt

Orians W, Reisach U (2017) Wissenstransfer in der Krankenund Altenpflege: Möglichkeiten und Grenzen der Digitalisierung von Wissen. In: Pfannstiel MA, Krammer S, Swoboda W (Hrsg) Digitale Transformation von Dienstleistungen im Gesundheitswesen III. Impulse für das Management. Springer Fachmedien, Wiesbaden, S 33-54

Ploch U, Werkmeister T (2017) Digitalisierung und Technisierung der Pflege in Deutschland. Aktuelle Trends und 
ihre Folgewirkungen auf Arbeitsorganisation, Beschäftigung und Qualifizierung. DAA-Stiftung Bildung und Beruf, Hamburg

Remmers H (2015) Natürlichkeit und Künstlichkeit. Zur Analyse und Bewertung von Technik in der Pflege des Menschen. Technikfolgenabschätzung. Theorie und Praxis 24(2):11-20

Roland Berger $\mathrm{GmbH}$, Deutsches Institut für angewandte Pflegeforschung e.V., Philosophisch-Theologische Hochschule Vallendar PF, Lehrstuhl für Gemeindenahe Pflege (2017) ePflege. Informations- und Kommunikationstechnologie für die Pflege. Studie im Auftrag des Bundesministeriums für Gesundheit, Roland Berger $\mathrm{GmbH}$. Deutsches Institut für angewandte Pflegeforschung e. V., Philosophisch-Theologische Hochschule Vallendar PF, Lehrstuhl für Gemeindenahe Pflege, Berlin Vallendar Köln

Rösler U, Schmidt K, Merda M, Melzer M (2018) Digitalisierung in der Pflege. Wie intelligente Technologien die Arbeit professioneller Pflegender verändern. Initiative Neue Qualität der Arbeit INQA, Berlin

Sachverständigenrat zur Begutachtung der Entwicklung im Gesundheitswesen (2012) Wettbewerb an der Schnittstelle zwischen ambulanter und stationärer Gesundheitsversorgung. Sondergutachten 2012, Langfassung. Sachverständigenrat zur Begutachtung der Entwicklung im Gesundheitswesen, Berlin
Schröder L, Urban H-J (Hrsg) (2016) Digitale Arbeitswelt Trends und Anforderungen. Jahrbuch Gute Arbeit. Bund, Frankfurt

Simon M (2015) Unterbesetzung und Personalmehrbedarf im Pflegedienst der allgemeinen Krankenhäuser. Eine Schätzung auf Grundlage verfügbarer Daten. Hochschule Hannover Fakultät V - Diakonie, Gesundheit und Soziales, Hannover

Sowinski C, Kirchen-Peters S, Hielscher V (2013) Praxiserfahrungen zum Technikeinsatz in der Altenpflege, Kuratorium Deutsche Altershife. Institut für Sozialforschung und Sozialwirtschaft (iso) e. V., Köln Saarbrücken

Vincent JL, Creteur J (2017) The hospital of tomorrow in 10 points. Crit Care 21(1):93

Wibbeling S, Hintze M, Deiters W (2017) Krankenhaus 4.0 - Industrie $4.0 \mathrm{im}$ Gesundheitswesen, Positionspapier. Fraunhofer-Innovationszentrum für Logistik und IT, Healthcare Innovation, Dortmund

Wong CM, Wu SY, Ting WH, Ho KH, Tong LH, Cheung NT (2015) An Electronic Nursing Patient Care Plan Helps in Clinical Decision Support. Studies in Health Technology and Informatics 216:945

Zander B, Dobler L, Bäumler M, Busse R (2014) Implizite Rationierung von Pflegeleistungen in deutschen Akutkrankenhäusern - Ergebnisse der internationalen Pflegestudie RN4Cast. Gesundheitswesen 76(11):727734

Open Access Dieses Kapitel wird unter der Creative Commons Namensnennung 4.0 International Lizenz(http://creativecommons. org/licenses/by/4.0/deed.de) veröffentlicht, welche die Nutzung, Vervielfältigung, Bearbeitung, Verbreitung und Wiedergabe in jeglichem Medium und Format erlaubt, sofern Sie den/die ursprünglichen Autor(en) und die Quelle ordnungsgemäß nennen, einen Link zur Creative Commons Lizenz beifügen und angeben, ob Änderungen vorgenommen wurden.

Die in diesem Kapitel enthaltenen Bilder und sonstiges Drittmaterial unterliegen ebenfalls der genannten Creative Commons Lizenz, sofern sich aus der Abbildungslegende nichts anderes ergibt. Sofern das betreffende Material nicht unter der genannten Creative Commons Lizenz steht und die betreffende Handlung nicht nach gesetzlichen Vorschriften erlaubt ist, ist für die oben aufgeführten Weiterverwendungen des Materials die Einwilligung des jeweiligen Rechteinhabers einzuholen. 